\title{
Vorwort Heft 2-10
}

\author{
Hans-Christoph Grunau
}

Online publiziert: 11. Juni 2010

(C) Vieweg+Teubner und Deutsche Mathematiker-Vereinigung 2010

In ihrem Übersichtsartikel „An update on the Hirsch conjecture“ lassen Edward D. Kim und Francisco Santos die aktuelle Entwicklung zu einer Vermutung aus der Polytop-Geometrie Revue passieren. Warren D. Hirsch hat 1957 vermutet, dass man je zwei Ecken eines $d$-dimensionalen Polytops mit $n$ Facetten über maximal $n-d$ Kanten verbinden kann. Die Autoren erläutern zunächst den Hintergrund und die Bedeutung dieser Vermutung im Zusammenhang mit linearen Optimierungsproblemen und gehen dann auf bewiesene Spezialfälle, Zweifel an der Allgemeingültigkeit, Modifikationen und bekannte Schranken ein. Der vorliegende Beitrag ist ganz überblicksartig gehalten; Details kann man in einer ausführlicheren Version auf dem arXiv-Server finden. Während des letzten Korrekturgangs für dieses Heft erreicht mich die ganz aktuelle Nachricht von Francisco Santos, dass er ein Gegenbeispiel zur Hirsch-Vermutung gefunden hat. Genaueres finden Sie in einer ,note added in proof“ am Ende des Übersichtsartikels.

In seinem historischen Beitrag ,Some direct and remote relations of Gauss with Belgian mathematicians" beleuchtet Jean Mawhin zunächst den Besuch Adolphe Quetelets - eines späteren Sekretärs der königlichen Akademie von Brüssel - bei Gauß. Ein sich anschließender Briefwechsel wirft ein interessantes Licht auf Gauß und den Wissenschaftsbetrieb des 19. Jahrhunderts. Im zweiten Teil rekapituliert der Autor den Beweis und dessen Vorgeschichte des von Gauß vermuteten Primzahlsatzes durch den belgischen Mathematiker Charles-Jean de La Vallée Poussin. Schließlich wird an Hand von de La Vallée Poussins Vortrag aus dem Jahre 1939 zum 100. Geburtstag von Gauß' Werk zur Potentialtheorie dargelegt, wie intensiv dieses nachfolgende Wissenschaftlergenerationen inspiriert und geprägt hat.

H.-Ch. Grunau (凶)

Institut für Analysis und Numerik, Fakultät für Mathematik, Otto-von-Guericke-Universität, Postfach 4120, 39016 Magdeburg, Deutschland

e-mail: hans-christoph.grunau@ovgu.de 
Beim Durchblättern dieses Heftes wird Ihnen sofort ein neues Erscheinungsbild auffallen. Der Verlag Vieweg + Teubner und die DMV haben sich auf ein neues Produktionsverfahren verständigt, das für die Leserinnen und Leser des Jahresberichtes eine Reihe von Verbesserungen bringen wird. Der Jahresbericht wird Bestandteil von SpringerLink und damit unter den dort üblichen Bedingungen unmittelbar online verfügbar. Die DMV pflegt weiterhin ihre Webseiten zum Jahresbericht, auf denen für jedermann sofort die bibliographischen Informationen und längere Zusammenfassungen und nach Ablauf von zwei Jahren sogar die Originaldateien der Beiträge kostenfrei verfügbar sind. Der Verlag Vieweg + Teubner ist der DMV sehr entgegengekommen; gemeinsam hoffen wir, dass diese Verbesserungen Ihre Freude am und auf den Jahresbericht steigern werden. 